Cite this: Phys. Chem. Chem. Phys., 2013, 15,20252

Received 30th August 2013, Accepted 6th October 2013

DOI: $10.1039 /$ c3cp53667j

www.rsc.org/pccp

\title{
Collective librations of water molecules in the crystal lattice of rubidium bromide: experiment and simulation $\dagger$
}

\author{
L. M. Lepodise,* J. Horvat and R. A. Lewis
}

\begin{abstract}
Terahertz spectroscopy of $\mathrm{RbBr}$ reveals four prominent absorption lines at room temperature and a further 15 lines at $10 \mathrm{~K}$. Via density-functional-theory (DFT) numerical modelling using the PBEO hybrid GGA functional, all the absorptions are identified as correlated librations of water molecules in the $\mathrm{RbBr}$ lattice. Each libration mode is a combination of rocking, wagging and twisting motions of the water molecules. The number of libration lines and numerical modelling show that the $C_{2 v}$ symmetry of water in $\mathrm{RbBr}$ is broken. Our modelling shows that the distribution of libration amplitudes and phases for different water molecules in the $\mathrm{RbBr}$ unit cell varies greatly between the different modes. All librational lines red-shift with increasing temperature. The rate of change for most lines is in the range $60-90 \mathrm{MHz} \mathrm{K}^{-1}$ (or $(2-3) \times 10^{-3} \mathrm{~cm}^{-1} \mathrm{~K}^{-1}$ ). Two lines shift more rapidly with temperature, at rates of 240 and $300 \mathrm{MHz} \mathrm{K}^{-1}$ (or $(8$ and 10$) \times 10^{-3} \mathrm{~cm}^{-1} \mathrm{~K}^{-1}$ ), respectively. Furthermore, the temperature dependence of the linewidth distinguishes two groups of lines. For one group, with weak linear temperature dependence of linewidth, cubic anharmonic terms in the RbBr crystal field are significant. This group is mainly associated with fully symmetric correlated librations. For the second group, with strong non-linear temperature dependence of the linewidth, quartic anharmonic terms in the $\mathrm{RbBr}$ crystal field are significant. However, the distribution of libration amplitudes, as well as the type of libration modes, influence the temperature dependence of the red shift, the linewidth, and the intensity, as well. Our combined experimental and theoretical investigation confirms the necessity of obtaining lowtemperature data to observe all the calculated modes; moreover, the richness of detail in the temperature dependence of the data invites further modelling spanning a range of temperatures.
\end{abstract}

\section{Introduction}

Water in crystal structures can undergo hindered rotations (or librations), which are categorised as rocking (R), wagging (W) and twisting $(\mathrm{T})$ modes, representing hindered rotation around the $x, y$ and $z$-axes, respectively. ${ }^{1,2}$ The study of the librational modes of water in crystals can be used to elucidate the crystal fields and the interaction of water with the crystal ions. This interaction is still not well understood, even though it plays a key role in the formation of hydrated crystals and proteins. ${ }^{3-7}$ Understanding the mechanisms of adsorption of water on surfaces and absorption in crystals on the molecular level is an important goal for many disciplines. The classical self-consistent formulation of water libration for water molecules confined by hydrophobic

Institute for Superconducting and Electronic Materials and School of Physics, University of Wollongong, Wollongong, New South Wales, 2522, Australia. E-mail:lml533@uowmail.edu.au, jhorvat@uow.edu.au,roger@uow.edu.au $†$ Electronic supplementary information (ESI) available: Animation files showing the vibrations of water in the $\mathrm{RbBr}$ lattice, for the lines identified by numerical simulations (Fig. 9). Two modes (denoted 15a and 15b) correspond to the observed line 15. See DOI: 10.1039/c3cp53667j interfaces shows that internal dipolar fields become enhanced at the hydration layer, with large local fluctuations. ${ }^{8}$ This is the dominant mechanism for slaving protein processes by the surrounding solvent. The hydrogen bonding network of water at interfaces of liquids and solids is a complex structure, relevant for an array of phenomena. ${ }^{9}$ Water molecules may change little during the absorption-adsorption. However, water can undergo strong structural changes, for example, when adsorbed on silica clusters. ${ }^{10}$ Water on the surface of $\mathrm{Ru}$ forms flat lying clusters, optimising the $\mathrm{Ru}$-water interaction at the expense of the water-water interaction. ${ }^{11}$ The changes in the water molecules upon absorption-adsorption will affect their vibrational and librational absorption lines, which makes infrared spectroscopy an effective tool in studying the interaction of water with solid matter.

Alkali halides have convenient properties making them suitable for studying the interactions of water in the crystalline environment. They are hygroscopic, but have high transmittance over a wide range of frequencies and so are employed as spectrometer windows in the far-infrared/terahertz regions. ${ }^{12-15}$ Far-infrared, ${ }^{16}$ visible $^{17}$ and ultraviolet $^{18}$ transmission 
measurements have been made of alkali halides previously. Pure alkali halides do not exhibit absorption lines in the farinfrared region. ${ }^{16,19}$ Crystal or surface imperfections, colour centres, or impurities do not produce absorption lines in this region either. However, introducing water into an alkali halide ${ }^{20-22}$ (or any other halide $^{23}$ ) yields absorption lines in the spectral range 9-20 $\mathrm{THz}$ (300-700 $\left.\mathrm{cm}^{-1}\right)$. These have been interpreted as arising from librations of water. The librational spectra of alkali halides found in the literature do not provide high enough resolution to draw detailed conclusions about the interaction between water and its crystal surroundings, as well as between the water molecules themselves. A single water molecule in crystal surroundings gives only two librational lines if its $C_{2 \mathrm{v}}$ symmetry is retained in the crystal, because the twisting mode is not infrared-active under $C_{2 \mathrm{v}}$ symmetry. ${ }^{1,2}$ If the $C_{2 v}$ symmetry is broken in the crystal, three lines are obtained. In addition, correlations between the librational modes of nearby water molecules will produce new absorption lines. The interaction of water with crystalline fields will affect the linewidth, intensity and energy of the librational lines. Different terms of crystalline field can result in a specific temperature dependence of these lines. ${ }^{24}$

The scarcity of the reports giving detailed temperature dependence of the linewidth, energy and intensity of the librational modes of water in alkali halides is addressed in this paper. High-resolution librational spectra of water in $\mathrm{RbBr}$ measured between 10 and $300 \mathrm{~K}$ reveal numerous librational modes and their temperature dependence for the first time. Further analysis of these experimental results through hybrid DFT modelling provides insights into the symmetry of water molecules in $\mathrm{RbBr}$ and the interactions between the librational modes and potential energies of the system.

\section{Procedure}

Several samples were cut from a crystalline RbBr infrared window, which was exposed to the atmospheric moisture over many years. Most detailed measurements were performed on a $5.6 \mathrm{~mm}$-thick $\mathrm{RbBr}$ sample, which is the focus of this report. The samples were characterised using X-ray diffraction measurements on a Philips Model PW1830 diffractometer employing $\mathrm{Cu}-\mathrm{K}_{\alpha 1}$ radiation $(0.154056 \mathrm{~nm})$. Samples were stationary during the XRD scans. A Mettler Toledo TGA/DSC1 thermogravimetric analyser, coupled to a Pfeiffer Thermostar GSD320 mass spectrometer, was used to assess the amount of water in the RbBr. Scanning electron microscopy (SEM) and energy-dispersive X-ray spectroscopy (EDS) measurements were taken using a JEOL 6490 LVSEM microscope equipped with a minicup EDS detector. The optical transmission was measured using a Fourier Transform Infrared Spectrometer (Bomem DA8). The source of radiation was a globar. For the lowfrequency region, $14-24 \mathrm{THz}\left(450-800 \mathrm{~cm}^{-1}\right)$, a coated, broadband beam splitter was employed and the detector was a liquid-heliumcooled bolometer. For the high-frequency region, 20-120 THz (650-4000 $\mathrm{cm}^{-1}$ ), a $\mathrm{KBr}$ beam splitter was employed and the detector was a liquid-nitrogen-cooled $\mathrm{HgCdTe}$ element. The resolution at all frequencies was $0.015 \mathrm{THz}\left(0.5 \mathrm{~cm}^{-1}\right)$. A reference spectrum, with no sample in the beam, was also collected. The ratio of the spectrum collected with the sample

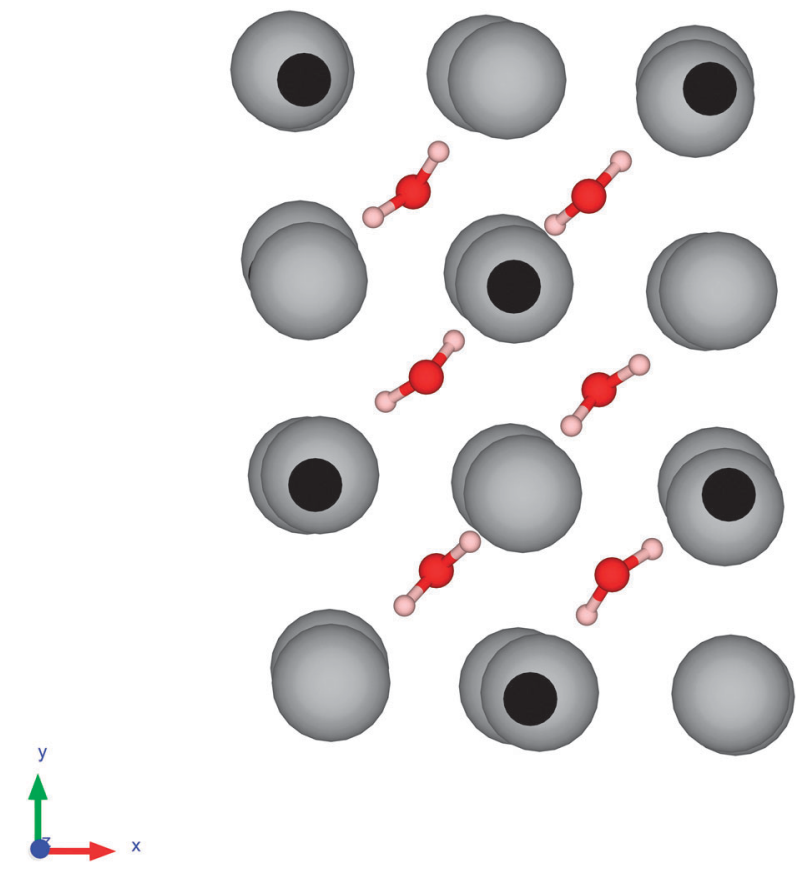

Fig. 1 Optimised geometry for modelling 6 water molecules in an RbBr crystal lattice using the PBEO method, showing the $(x, y)$ face of the crystal. The small dark spheres are bromine and large grey spheres are rubidium atoms. ${ }^{33}$

in beam path to the reference spectrum gave the transmission spectrum. The sample was cooled in a continuous-flow helium cryostat to obtain low-temperature spectra.

Numerical modelling was performed using the Orca ab-initio, DFT and semi-empirical electronic structure package. ${ }^{25}$ The PerdewBurke-Ernzerhof (PBE) gradient-corrected density functional ${ }^{26}$ and its hybrid form $\mathrm{PBE0}^{27}$ were used, and an empirical Van der Waals correction was employed. ${ }^{28}$ An Ahlrichs TZVPP++ basis set was used in all calculations. ${ }^{29-32}$ The $\mathrm{RbBr}$ lattice consisted of $4 \times 4 \times 3$ atoms (see Fig. 1), with 1 to 6 water molecules added to the interstitial cavities of the middle layer.

Modelling more than 6 water molecules would require additional layers and be impractically time-consuming. Keeping the water molecules in the middle layer (Fig. 2) stabilised the RbBr lattice against distortions that might occur as a consequence of the relatively small number of atoms used in the model. Absorption frequencies were calculated in the harmonic approximation without thermal effects, after tight-geometry optimisation. PBE and PBE0 functionals are suitable for modelling solids, being correct in the uniform electron density limit. ${ }^{34}$ The PBE0 method includes $25 \%$ of the Hartree-Fock exchange interaction and so is more accurate than the PBE method, but is significantly more time-consuming. Consequently, PBE0 was only used in the simulation with 6 water molecules.

\section{Results and discussion}

The room-temperature spectrum of $\mathrm{RbBr}$, displaying the water libration modes, is given in the top part of Fig. 3. Four prominent absorption lines can be identified, at 17.62, 18.26, 18.68 and 


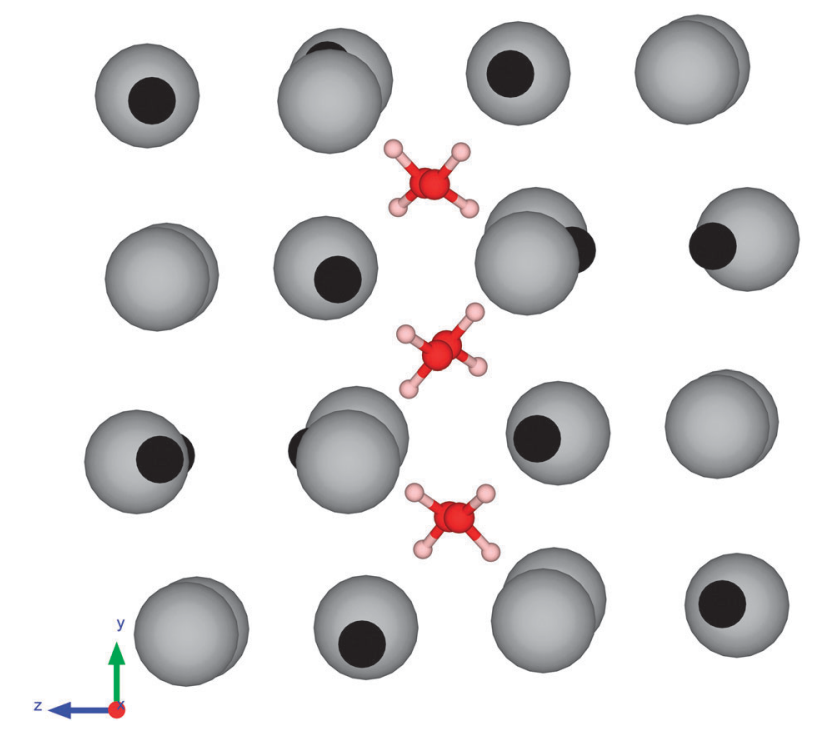

Fig. 2 Optimised geometry for modelling 6 water molecules in an RbBr crystal lattice using the PBEO method, showing the $(y, z)$ face of the crystal. The small dark spheres are bromine and large grey spheres are rubidium atoms. ${ }^{33}$

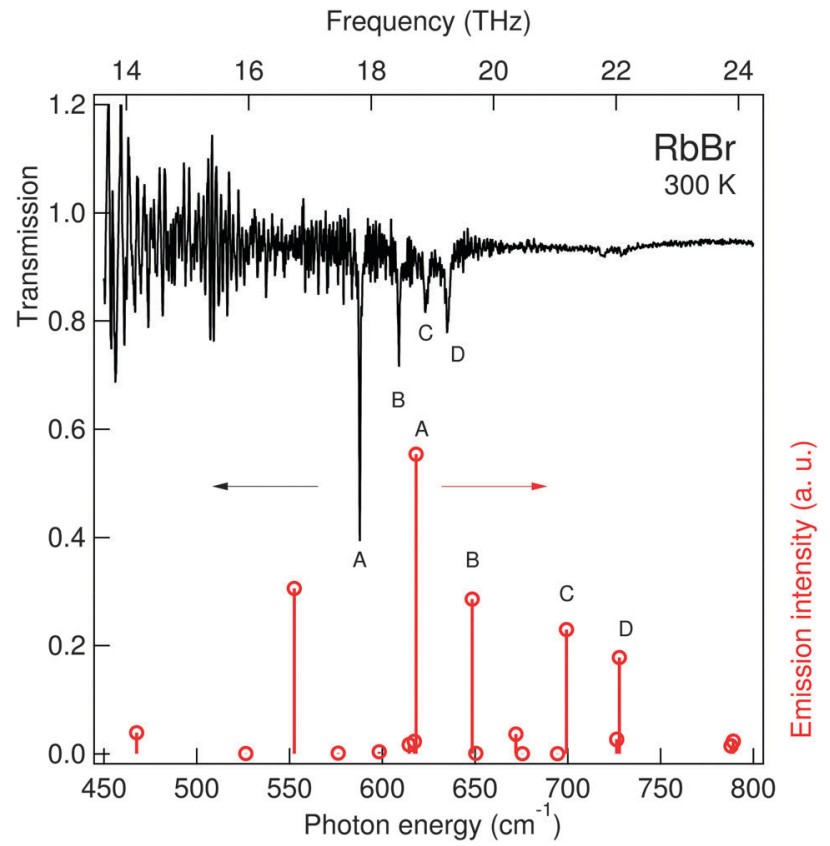

Fig. 3 Libration spectrum of water in $\mathrm{RbBr}$ observed at room temperature. The top (black) curve is the experimental transmission spectrum and the bottom lines (red) give the energies and relative intensities of the emission spectrum, calculated by the PBEO method for 6 water molecules in the RbBr lattice (Fig. 1 and 2).

19.03 THz (587.89, 608.98, 623.14 and $\left.634.93 \mathrm{~cm}^{-1}\right)$. These are labelled A-D, respectively. Water libration modes for water in alkali halides, ${ }^{20-22}$ liquid water, water ice, ${ }^{35}$ water on metal oxide surfaces $^{36}$ and water confined in rigid pores ${ }^{37}$ are reported at these energies. Numerical modelling was performed to verify that the observed absorption lines are indeed due to librations of water. The modelling using the PBE0 method with 6 molecules of water in the $\mathrm{RbBr}$ lattice gave a very good agreement with

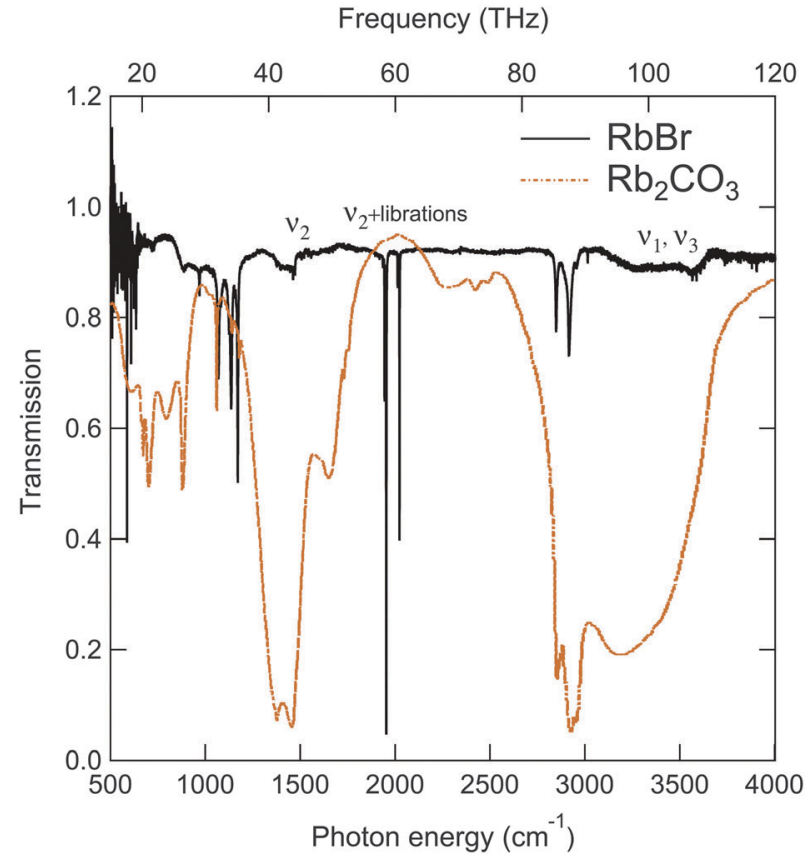

Fig. 4 Infrared spectrum of $\mathrm{RbBr}$ between 15 and $120 \mathrm{THz}$ (500 and $\left.4000 \mathrm{~cm}^{-1}\right)$, together with the $\mathrm{Rb}_{2} \mathrm{CO}_{3}$ spectrum ${ }^{38}$ for comparison. The labels $\nu_{1}, \nu_{2}$ and $\nu_{3}$ indicate water vapour vibrational modes and $\nu_{2}+$ librations indicates a presumed interaction between the $\nu_{2}$ vibrational and the librational modes at higher frequency.

these four experimental absorption lines, considering the relatively low frequencies involved. The four observed lines at room temperature (A-D) are recognisable in the numerical spectrum, albeit shifted to higher energies. The lines calculated at 14.0 and 16.6 $\mathrm{THz}$ (468 and $553 \mathrm{~cm}^{-1}$ ) were not observed at room temperature, but can be identified in low-temperature spectra, as more lines appear at low temperatures.

The $\mathrm{RbBr}$ spectrum at higher frequencies, up to $120 \mathrm{THz}$ $\left(4000 \mathrm{~cm}^{-1}\right)$, is shown in Fig. 4 . Vibrational absorption lines of free water are expected in this region: bending $\nu_{2}$ lines at around $48 \mathrm{THz}\left(1600 \mathrm{~cm}^{-1}\right)$; symmetric stretching $\nu_{1}$ lines at around $108 \mathrm{THz}\left(3600 \mathrm{~cm}^{-1}\right)$; and asymmetric stretching $\nu_{3}$ lines at around $114 \mathrm{THz}\left(3800 \mathrm{~cm}^{-1}\right)$. Measurements of vibrational lines for water in a number of alkali halides ${ }^{39}$ show that the energy of these lines is affected by crystalline fields. The $\nu_{3}$ line of water in $\mathrm{RbBr}$ was reported at $103 \mathrm{THz}\left(3425 \mathrm{~cm}^{-1}\right) .{ }^{39}$ It is shifted by $175 \mathrm{~cm}^{-1}$ in $\mathrm{RbBr}$ crystalline fields. We observe two absorption bands at these frequencies, centred at 97.5 and $106.5 \mathrm{THz}(3254$ and $3553 \mathrm{~cm}^{-1}$ ), which correspond to $\nu_{1}$ and $\nu_{3}$ vibrational lines, respectively. The positions of these vibrational water lines is identified in Fig. 4. The observed strong lines around $60 \mathrm{THz}$ $\left(2000 \mathrm{~cm}^{-1}\right)$ are remarkable in that the $\nu_{2}$ bending modes of free water molecules are expected to be at much lower frequency, at around $48 \mathrm{THz}\left(1600 \mathrm{~cm}^{-1}\right)$. It appears the $\nu_{2}$ bending modes of water are shifted to higher frequencies as a result of interactions with the $\mathrm{RbBr}$ ions and through the correlation of vibrational and librational modes of water. ${ }^{40}$

Vibrational lines of water in $\mathrm{CsCl}$ are reported at 2110 and $1997 \mathrm{~cm}^{-1}$ for large water density, while in water ice they appear 
at 2243 and $2041 \mathrm{~cm}^{-1}$. ${ }^{39}$ Water itself in the liquid state gives absorption lines around $60 \mathrm{THz}\left(2000 \mathrm{~cm}^{-1}\right)$, which is explained as the interaction between the $\nu_{2}$ bending mode of water and its librational modes. ${ }^{41,42}$ Our measurements give two sets of lines: one set consists of lines at 1953, 1944 and a weak one at $1935 \mathrm{~cm}^{-1}$, while the other set consists of two lines at 2013 and $2023 \mathrm{~cm}^{-1}$. The latter set was of lower intensity than the former. The splitting within each set of the lines probably occurred because of water agglomeration into clusters with different water concentration, as shown by splitting of (h00) XRD peaks for RbBr into 3 sub-peaks (Fig. 6). These lines are unexpectedly strong (relative to $\nu_{1}$ and $\nu_{3}$ lines of water), and consequently this interpretation should be treated with caution. However, no other compound absorbing at these energies could be identified. These shifts of $\nu_{2}$ lines to higher energies can only occur if anharmonic effects are present, ${ }^{43}$ which would explain why they are not obtained in our numerical modelling using a harmonic approximation.

While the three vibrational modes of water account for some of the features in the spectrum, many other lines remain. Another source for these lines must be found. EDS analysis of the $\mathrm{RbBr}$ samples revealed the presence not only of $\mathrm{Rb}$ and $\mathrm{Br}$, but also of $\mathrm{C}$ and $\mathrm{O}$. Since $\mathrm{RbBr}$ is typically synthesised from $\mathrm{HBr}$ and $\mathrm{Rb}_{2} \mathrm{CO}_{3}$, it was speculated that the additional peaks may be due to some residual $\mathrm{Rb}_{2} \mathrm{CO}_{3}$. Indeed, good agreement was obtained between the $\mathrm{Rb}_{2} \mathrm{CO}_{3}$ spectrum ${ }^{38}$ and the spectrum of our samples for the absorption lines not belonging to water, occurring at 712, 879, 1063, 1135, 1171, 1374, 1448, 2848 and $2915 \mathrm{~cm}^{-1}$ (Fig. 4). In comparing the two spectra further, the $\mathrm{Rb}_{2} \mathrm{CO}_{3}$ spectrum does not have absorption lines at energies where the librational lines of water occur.

The distinct groups of absorption lines in the $\mathrm{RbBr}$ sample are all well separated from each other. This segregation is also reflected in the numerical modelling. The lattice vibrations of the $\mathrm{RbBr}$ lattice occur between 1 and $8 \mathrm{THz}\left(35\right.$ and $270 \mathrm{~cm}^{-1}$ ), according to our PBE0 calculations. The librational modes of water obtained with the same method were between 14 and $24 \mathrm{THz}$ (467 and $789 \mathrm{~cm}^{-1}$ ). The bending vibration lines of water, $\nu_{2}$, were between 49.7 and $50.2 \mathrm{THz}\left(1657\right.$ and $1676 \mathrm{~cm}^{-1}$ ). The stretching vibration lines, $\nu_{1}$ and $\nu_{3}$, were between 104.7 and 109.6 THz ( 3478 and $3655 \mathrm{~cm}^{-1}$ ). The modelling showed that all the vibrational lines were correlated between neighbouring water molecules in the crystal lattice, resulting in a line splitting. For all librational and vibrational modes of water, the $\mathrm{Rb}$ and $\mathrm{Br}$ ions were stationary. Taken together, this information permits an unambiguous identification of the observed absorption lines around $18 \mathrm{THz}\left(600 \mathrm{~cm}^{-1}\right)$ as being due to the correlated librations of water molecules in $\mathrm{RbBr}$. The ESI $\dagger$ provides visualisation files for different libration modes. Many of the calculated libration modes were of low intensity and consequently were not observed in the experiment.

Thermogravimetric analysis was performed on a pulverised sample that gave the IR spectrum as in Fig. 3 and 4, by heating the sample to $500{ }^{\circ} \mathrm{C}$ over 2 hours. The sample was pulverised because infrared windows are made so that they do not absorb or release water easily and water penetrates pure, defect-free crystals of alkali halides very slowly. ${ }^{44}$ Mass spectrometery coupled to the thermogravimetric chamber showed that surface water was released from the sample up to $200{ }^{\circ} \mathrm{C}$, followed by a slower release of volume water at higher temperatures. The surface water was adsorbed to the crystallites during the sample handling. We estimate there was 1 water molecule per $14 \mathrm{RbBr}$ molecules in the volume of the crystallites. The pulverised sample absorbed water easily and the infrared absorption lines were broadened. The unusual lines around $2000 \mathrm{~cm}^{-1}$ were no longer observed after the pulverisation of the sample. To further confirm the observed absorption lines are due to water, measurements were also performed on a $\mathrm{RbBr}$ sample cut from a fresh infrared window. Because this window was produced recently, its exposure to the atmospheric moisture was minimal. The librational and vibrational lines of water were not present for this sample. This supports the interpretation of the lines around $600 \mathrm{~cm}^{-1}$ as the librational lines of water. The strong lines around $2000 \mathrm{~cm}^{-1}$ were not observed for this sample.

The XRD pattern of a RbBr sample is shown in Fig. 5. $\mathrm{RbBr}$ crystallises in the face-centred cubic structure, space group $F m \overline{3} m$. Comparison to the published data for the RbBr XRD spectrum (Card No. 08-0480) confirms that the main XRD peaks belong to $\mathrm{RbBr}$. The two larger $\mathrm{RbBr}$ peaks belong to ( $h 00)$, while the two smaller peaks belong to $(h k 0)$ planes. Two expected $(h k l)$ peaks in this range of $2 \theta,(222)$ and (422), were not observed. The missing peaks show that the sample did not consist of large number of randomly oriented crystallites, but of a small number of single crystals. All expected XRD peaks for $\mathrm{RbBr}$ were obtained when the sample was ground into a powder and re-measured.

The (200) and (400) peaks are split into three sub-peaks, as shown in detail in Fig. 6. This splitting suggests agglomeration of water in the RbBr crystals into clusters with different water density and a consequent change of the lattice parameter. The values of the obtained lattice parameters were $0.686,0.688$ and $0.694 \mathrm{~nm}$ for XRD peak (200) and $0.687,0.689$ and $0.692 \mathrm{~nm}$ for

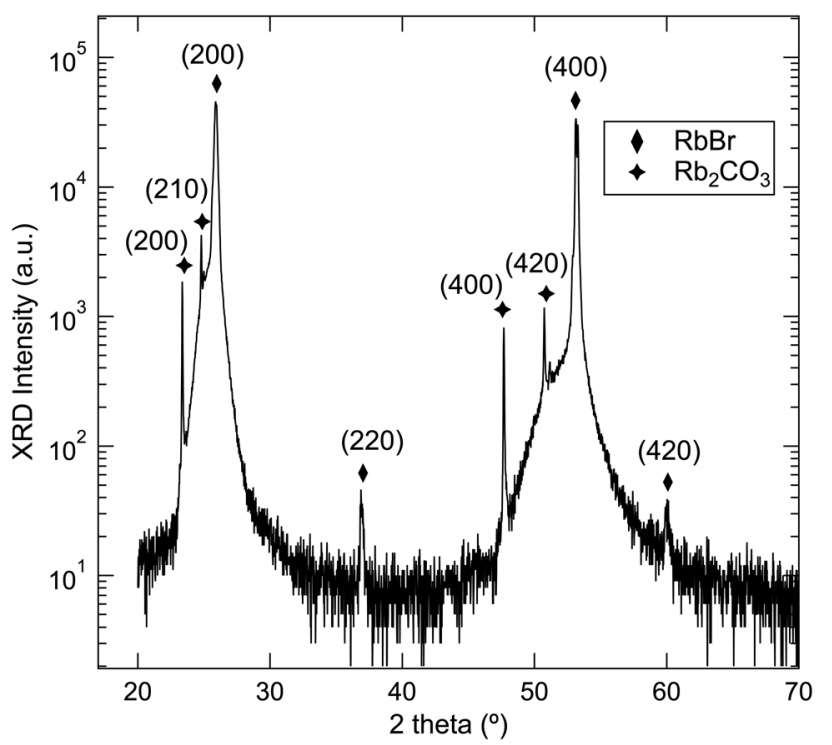

Fig. 5 XRD pattern of $\mathrm{RbBr}$ sample. Reflection planes of both $\mathrm{RbBr}$ and $\mathrm{Rb}_{2} \mathrm{CO}_{3}$ are indicated. 


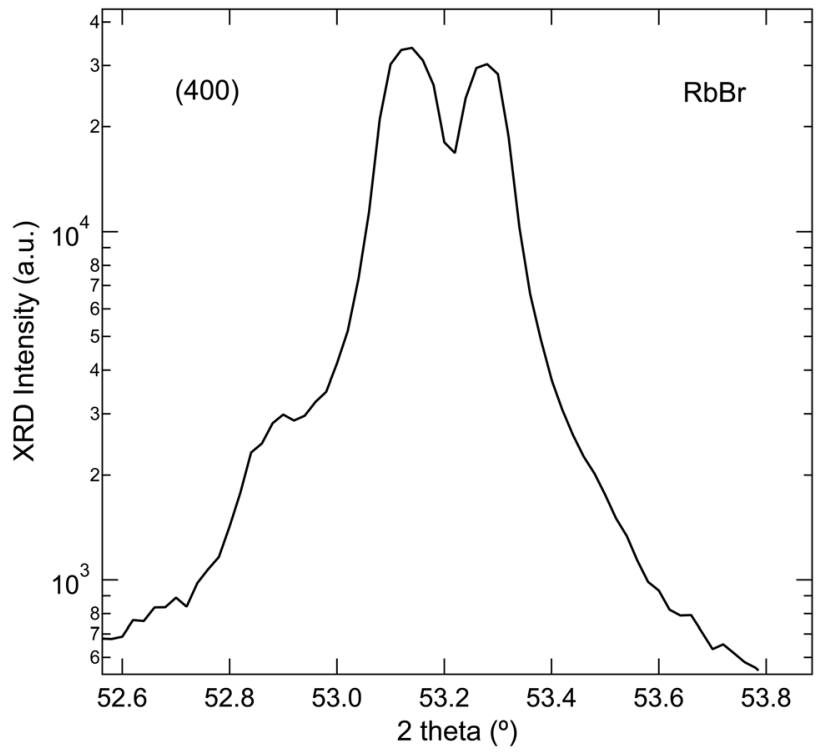

Fig. 6 Fine detail of the XRD pattern of peak (400) for $\mathrm{RbBr}$, showing splitting of the peak.

XRD peak (400). PBE0 modelling with 6 water molecules gave a fairly distorted structure due to the small number of atoms used, with lattice parameter between 0.691 and $0.707 \mathrm{~nm}$. XRD measurements for the powdered sample before and after its heat treatment at $500{ }^{\circ} \mathrm{C}$ for 2 hours show that this drying shifts the (400) peak by $0.1^{\circ}$. The remaining small XRD peaks can be identified as $\mathrm{Rb}_{2} \mathrm{CO}_{3}$ peaks, belonging to the (h00) and ( $\left.h k 0\right)$ planes (Card No. 35-0973). Thus XRD analysis confirms that there is residual $\mathrm{Rb}_{2} \mathrm{CO}_{3}$ in our samples, supporting the conclusions already put forward based on EDS and optical measurements.

Low-temperature measurements were made in the range 10-250 $\mathrm{K}$ and are shown in Fig. 7. Further detail at lower temperatures is shown in Fig. 8. In addition to the four lines observed at room temperature (A-D), fifteen new lines are observed, marked in the figure as 1-15. Lines $2,3,4,5,6,12$, and 13 are difficult to see, but can be distinguished from the background variations of the optical transmission by monitoring the change of the spectrum with temperature. Only the features in the spectrum which shifted with temperature were assigned as absorption lines. This distinguished them from the noise and background oscillations in the spectrum occurring because of multiple reflections in the optical path. The positions, linewidths and relative intensities of all observed absorption lines at $10 \mathrm{~K}$ are given in Table 1.

The total number of observed libration lines is 19 . There can be up to 6 nearest neighbours for each water molecule in the $\mathrm{RbBr}$ structure, defined by the cluster with the largest concentration of water. The interaction between the water molecules leads to the loss of degeneracy for the librational modes of water. This implies that more than two libration lines per water molecule are observed in our experiment, taking into account only the nearest-neighbour interactions. The twisting libration mode does not produce optical absorption lines under the $C_{2 \mathrm{v}}$ symmetry of water. $^{1,2}$ Thus 14 absorption lines should result in interaction

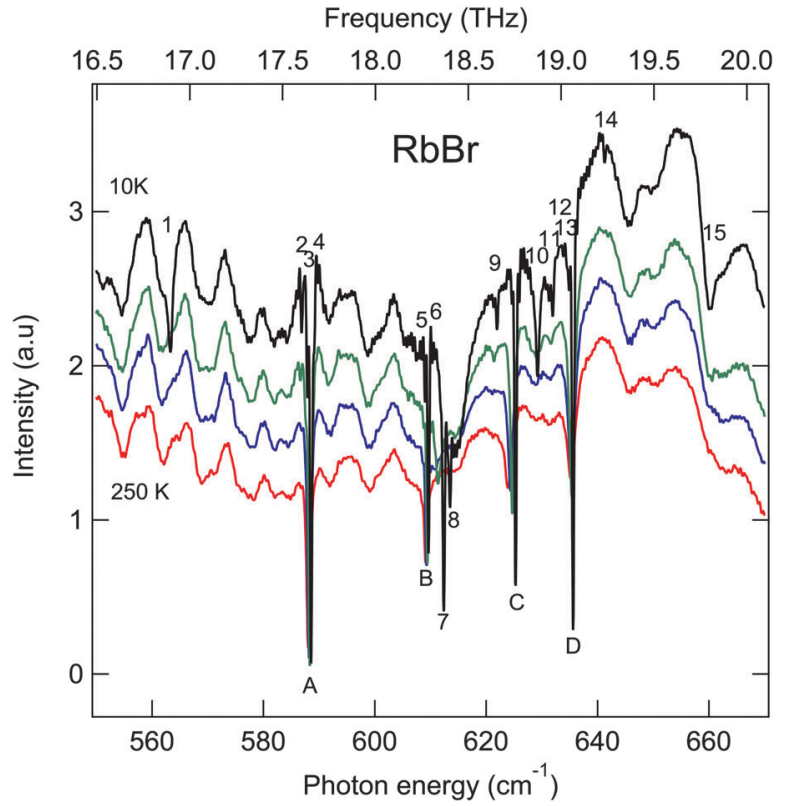

Fig. 7 Absorption spectrum for libration modes of water measured at 10, 150, 200 and $250 \mathrm{~K}$ shown at four temperatures.

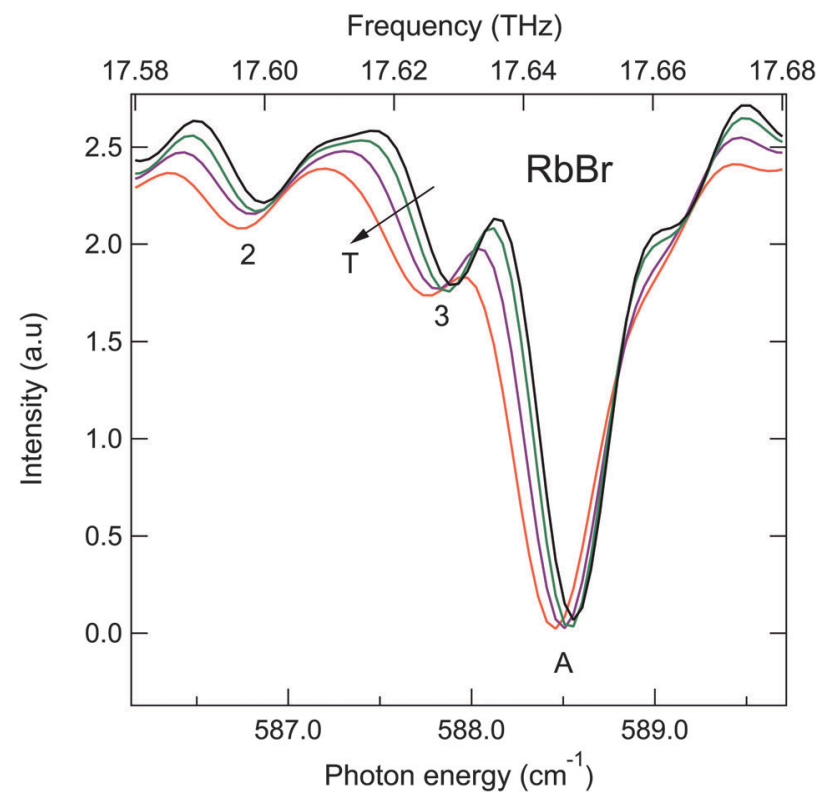

Fig. 8 Detail of the absorption lines 2, 3 and $A$ at 100, 75, 50 and $10 \mathrm{~K}$.

of the central water molecule with its 6 nearest neighbours under the $C_{2 \mathrm{v}}$ symmetry. Since we observe more than 14 lines, we conclude that the $C_{2 \mathrm{v}}$ symmetry of water is broken in the $\mathrm{RbBr}$ crystal. Given the broken $C_{2 \mathrm{v}}$ symmetry, 21 absorption lines are now possible. The missing 2 lines are either merged with the observed peaks or they are hidden in the experimental noise. Neutron diffraction studies have shown that water molecules in crystal hydrates have broken $C_{2 \mathrm{v}}$ symmetry in about $80 \%$ of the cases. ${ }^{45}$

Modelling with the PBE0 method gave 18 absorption lines between 14 and $24 \mathrm{THz}$ (467 and $789 \mathrm{~cm}^{-1}$ ) with 6 waters in the 
Table 1 Main properties of water libration lines deduced from the observed spectra at the lowest temperature measured, $10 \mathrm{~K}$

\begin{tabular}{|c|c|c|c|c|c|}
\hline \multirow[b]{2}{*}{ Label } & \multicolumn{2}{|l|}{ Position } & \multicolumn{2}{|l|}{ Linewidth } & \multirow[b]{2}{*}{$\begin{array}{l}\text { Intensity } \\
\text { (relative) }\end{array}$} \\
\hline & $\begin{array}{l}\text { Frequency } \\
(\mathrm{THz})\end{array}$ & $\begin{array}{l}\text { Energy } \\
\left(\mathrm{cm}^{-1}\right)\end{array}$ & $\begin{array}{l}\text { Frequency } \\
(\mathrm{GHz})\end{array}$ & $\begin{array}{l}\text { Energy } \\
\left(\mathrm{cm}^{-1}\right)\end{array}$ & \\
\hline 1 & 16.89 & 563.30 & 80.6 & 2.69 & 0.35 \\
\hline 2 & 17.59 & 586.87 & 9.3 & 0.31 & 0.31 \\
\hline 3 & 17.63 & 587.93 & 16.8 & 0.56 & 0.33 \\
\hline A & 17.64 & 588.55 & 13.2 & 0.44 & 2.53 \\
\hline 4 & 17.68 & 589.76 & 5.7 & 0.19 & 0.13 \\
\hline 5 & 18.26 & 609.03 & 10.5 & 0.35 & 0.31 \\
\hline B & 18.28 & 609.65 & 8.4 & 0.28 & 1.45 \\
\hline 6 & 18.30 & 610.30 & 7.2 & 0.24 & 0.14 \\
\hline 7 & 18.36 & 612.37 & 15.0 & 0.50 & 1.23 \\
\hline 8 & 18.39 & 613.48 & 10.8 & 0.36 & 0.55 \\
\hline 9 & 18.64 & 621.92 & 9.0 & 0.30 & 0.21 \\
\hline $\mathrm{C}$ & 18.74 & 625.25 & 9.3 & 0.31 & 2.16 \\
\hline 10 & 18.86 & 629.20 & 24.0 & 0.80 & 0.54 \\
\hline 11 & 18.95 & 631.95 & 6.0 & 0.20 & 0.21 \\
\hline 12 & 19.00 & 633.88 & 6.3 & 0.21 & 0.09 \\
\hline 13 & 19.03 & 634.89 & 8.4 & 0.28 & 0.16 \\
\hline $\mathrm{D}$ & 19.06 & 635.61 & 10.2 & 0.34 & 2.79 \\
\hline 14 & 19.22 & 641.25 & 8.7 & 0.29 & 0.13 \\
\hline 15 & 19.79 & 660.15 & 149.9 & 5.00 & 0.20 \\
\hline
\end{tabular}

$\mathrm{RbBr}$ lattice. The general shape of the calculated libration spectrum resembles the measured spectrum quite well, however the calculated lines are shifted by up to $1.5 \mathrm{THz}\left(50 \mathrm{~cm}^{-1}\right)$ from the corresponding measured lines. Frequency shifts of this magnitude commonly occur in DFT modelling, due to approximations used in the theory, basis sets, geometry optimisation and numerical errors. The harmonic approximation used in calculating the vibrational frequencies tends to overestimate the frequencies. ${ }^{46}$ The use of universal scale factors has been investigated to correct for the harmonic approximation. This scaling gave numerical predictions to within $3 \%$ from the experimental values for a number of compounds. ${ }^{47}$ However, simple scaling was inadequate for vibrations with strong anharmonic effects. To help match the calculated with observed absorption lines at $10 \mathrm{~K}$, the PBE0 energies were first divided by a factor of 2.4 to narrow the energy range of the lines and then $10 \mathrm{THz}\left(333 \mathrm{~cm}^{-1}\right)$ was added to align the spectrum with the measured lines. The purpose of this manipulation is solely to help in identification of the absorption lines, with the unscaled numerical frequencies shown in Fig. 3. Seven lines give a clear match between the experiment and modelling, as indicated in Fig. 9, where the scaled values are displayed. The calculated energy of line A is $5 \%$ higher than observed (Fig. 3).

Analysis of the dynamics of the modelled atoms shows that all the calculated lines are produced by correlated librations of water molecules within the $\mathrm{RbBr}$ lattice, the $\mathrm{Rb}$ and $\mathrm{Br}$ atoms remaining stationary. The faster PBE method was also utilised for different numbers of water molecules in the $\mathrm{RbBr}$ cell $(N=1$, 2, 4 and 6). This method gave absorption lines in the same energy range as PBE0, however the agreement with the experiment was significantly poorer in terms of both intensity and energy of the peaks. The number of correlated librational modes of water using this modelling was found to be $3 N$. In addition to this, $N$ modes of correlated water molecule vibrations were obtained at energies around $48 \mathrm{THz}\left(1600 \mathrm{~cm}^{-1}\right)$,

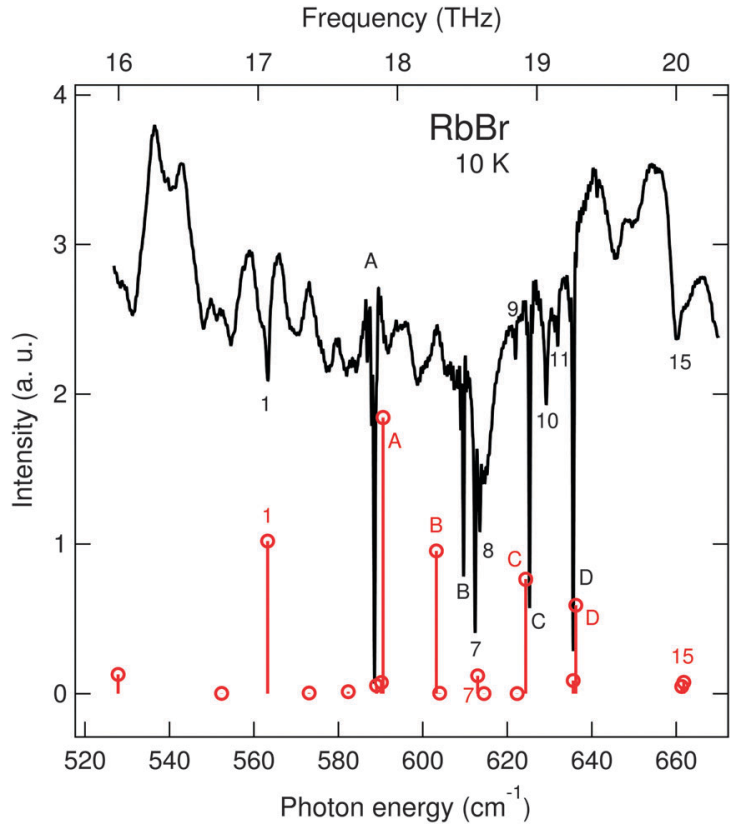

Fig. 9 Libration lines measured at $10 \mathrm{~K}$ (top) compared with the lines calculated using the PBEO method (bottom). The calculated lines were scaled to help identify the libration modes corresponding to each line. The true positions of the calculated lines are in Fig. 3.

corresponding to the bending $\nu_{2}$ mode of free water. Moreover, $2 \mathrm{~N}$ correlated vibrational modes of water were obtained around $105 \mathrm{THz}\left(3500 \mathrm{~cm}^{-1}\right)$, corresponding to correlated stretching $\nu_{1}$ and $\nu_{3}$ modes. This is as expected for the correlated water vibrations in $\mathrm{RbBr}$, demonstrating that the PBE modelling captures the essential underlying physics. Absorption lines due to vibrations of $\mathrm{RbBr}$ ions were found below $9 \mathrm{THz}\left(300 \mathrm{~cm}^{-1}\right)$. The $\mathrm{H}-\mathrm{O}-\mathrm{H}$ angle obtained by averaging over all 6 water molecules in $\mathrm{RbBr}$ using the PBE0 method was $105.474^{\circ}$, with an average O-H distance of $0.0974 \mathrm{~nm}$. The same method gave the value of this angle for a free water molecule as $105.00^{\circ}$, with $\mathrm{O}-\mathrm{H}$ distance $0.0958 \mathrm{~nm}$, again implying that the $C_{2 \mathrm{v}}$ symmetry for water in $\mathrm{RbBr}$ was broken. The experiment for free water gives the $\mathrm{O}-\mathrm{H}$ distance of $0.095762 \mathrm{~nm}$ and the $\mathrm{H}-\mathrm{O}-\mathrm{H}$ angle as $104.51^{\circ} .{ }^{48}$

Correlation between the librational modes of water is the key mechanism for obtaining the $3 N$ absorption lines. This correlation depends on the distance between the water molecules and the ions in the RbBr lattice, as well as on the distance between the water molecules themselves. In the PBE0 optimised geometry, the average distance between the water oxygens was $(0.296 \pm 0.003)$ $\mathrm{nm}$ in the $(x, y)$ plane (Fig. 1$)$ and $(0.321 \pm 0.007) \mathrm{nm}$ along the $z$-axis (Fig. 2). The water hydrogens pointed towards the $\mathrm{Br}$ atoms and the average distance between the water hydrogens and $\mathrm{Br}$ was $(0.225 \pm 0.005) \mathrm{nm}$. The librational correlations between the water molecules at these distances are in agreement with the studies of correlation effects between angular jumps of water molecules in liquid water. ${ }^{49}$ The correlation between the water molecule jumps was strongest at $0.35 \mathrm{~nm}$, where the minimum in the radial distribution function of water was found. Substantial correlations were obtained at distances up to $0.6 \mathrm{~nm}$. Study of molecular dynamics of liquid water shows that the processes 


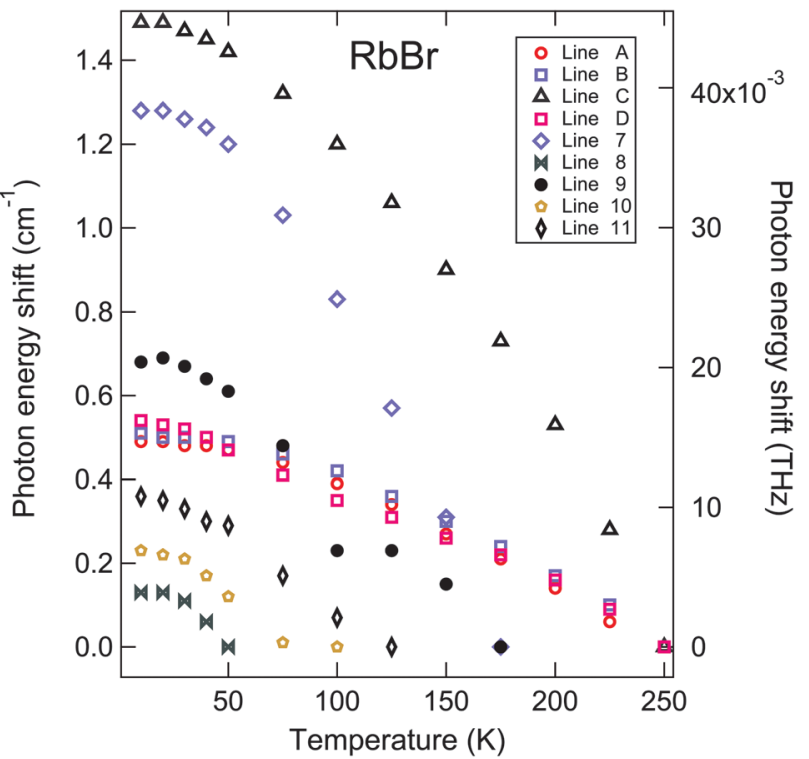

Fig. 10 Change in the line frequency with temperature, for several characteristic water lines. The line shift is calculated relative to the position at which each line appears for the highest temperature it is observed.

occurring on pico-second to 50 fs timescales are inherently collective. ${ }^{50}$ All this is in agreement with our conclusion that the librations of water in $\mathrm{RbBr}$ are of a collective nature.

Fig. 10 shows the observed shift of the libration lines with temperature. Only selected sets of lines are shown for clarity. Most of the lines shift little for $T<50 \mathrm{~K}$ but shift substantially at higher temperatures. The rate of the substantial energy shift with temperature for each line is given in Table 2, in the approximation of linear temperature dependence.

All but one of the lines red-shifts with temperature, which is usually associated with the increase in bond lengths upon warming. ${ }^{4}$
Line 15 gave an anomalous blue shift, which suggests it comprises two lines not resolved by our spectrometer. The weak temperature dependence of the line shift below $50 \mathrm{~K}$ suggests that the shift is dependent on phonon excitations of $\mathrm{RbBr}$ lattice, with most of the phonon modes being frozen-in at low temperatures. A group of libration lines can be identified that shift very weakly with temperature, between 60 and $90 \mathrm{MHz} \mathrm{K}{ }^{-1}$ (between (2 and 3) $\times 10^{-3} \mathrm{~cm}^{-1} \mathrm{~K}^{-1}$ ), lines 1, 2, 3, A, 5, B, 6, D. In contrast, lines 7 and $\mathrm{C}$ shift strongly.

Fig. 11 and 12 show the temperature dependence of the linewidth $\Gamma$, defined as the full width at half maximum (FWHM), for various libration lines. The linewidth increases with temperature for all lines. For absorption lines 1, 2, 3, A, 4, $8,9,10,11$ and $15, \Gamma$ is constant up to a given temperature, $T_{0}$, shown in Table 2 . The temperature dependence of $\Gamma$ can be described as:

$$
\Gamma(T)=\Gamma_{0}+a T+b T^{2},
$$

where $\Gamma_{0}$ is the "rest linewidth" and $T$ is the absolute temperature. ${ }^{1}$ The temperature dependence of $\Gamma$ has been studied theoretically for $\mathrm{NaCl}$ and $\mathrm{LiF}$ crystals. ${ }^{24}$ Linear and quadratic terms in the temperature dependence of $\Gamma$ were shown to arise from cubic and quartic terms in the crystal potential, respectively.

Absorption lines 2, A, 5, B, 6, 12, and 13 exhibit a weak $\Gamma(T)$ (Fig. 11), which is linear in temperature except for the line B. The quadratic term in eqn (1) is significant for the line B. These lines also shifted weakly with temperature (Table 2). Values of $\Gamma_{0}$ and $a$ for these lines are given in Table 2. The cubic anharmonic term in the crystal field is important for these librations. ${ }^{24}$ Absorption lines 1, 3, 7, 8, 9, 10, 11, 15, C, and D have a substantially stronger temperature variation in linewidth (Fig. 12). The non-linear $\Gamma(T)$ for these lines does not follow the exponential dependence on $1 / T$, as expected for systems where

Table 2 Changes with temperature of the main properties of water libration lines deduced from the observed spectra. The temperature below which the line width is almost constant is denoted $T_{0}$ (asterisked data are too noisy to make an accurate determination)

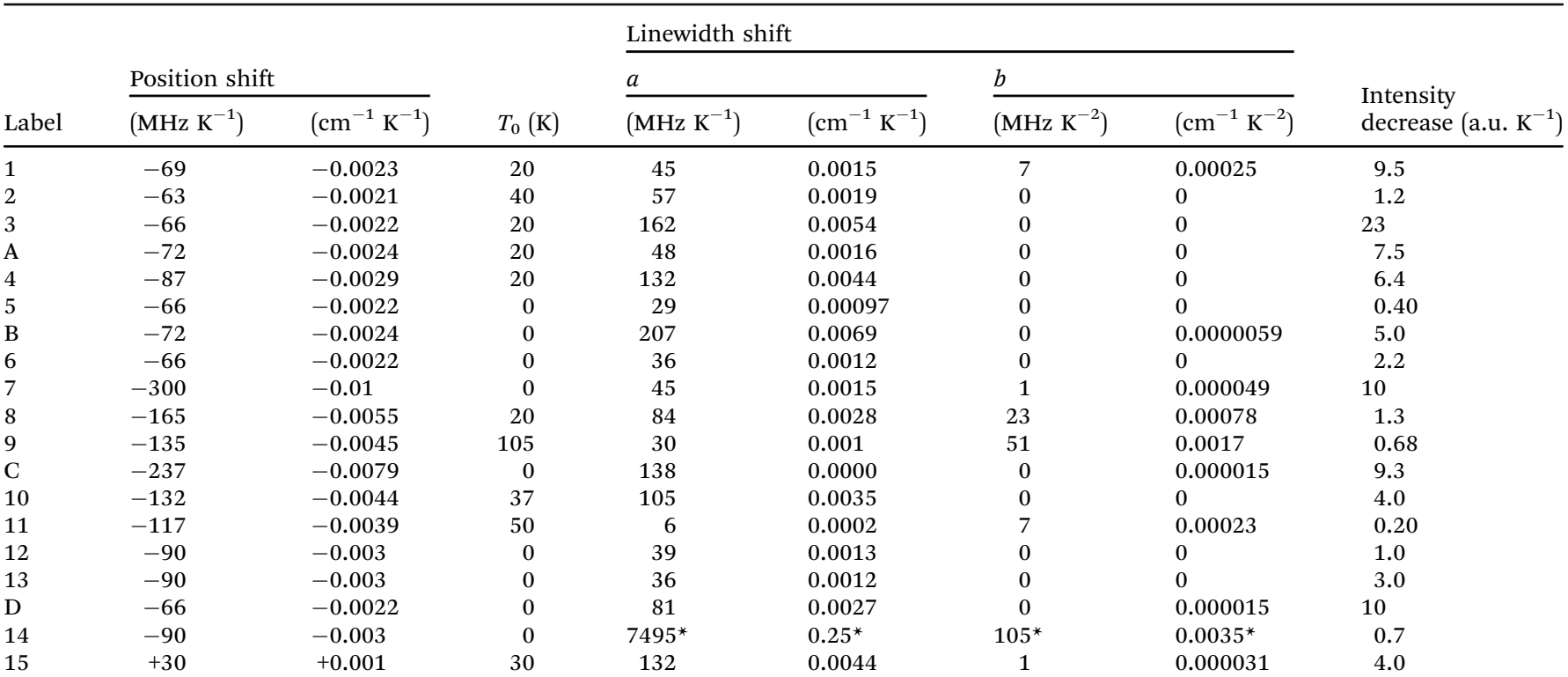




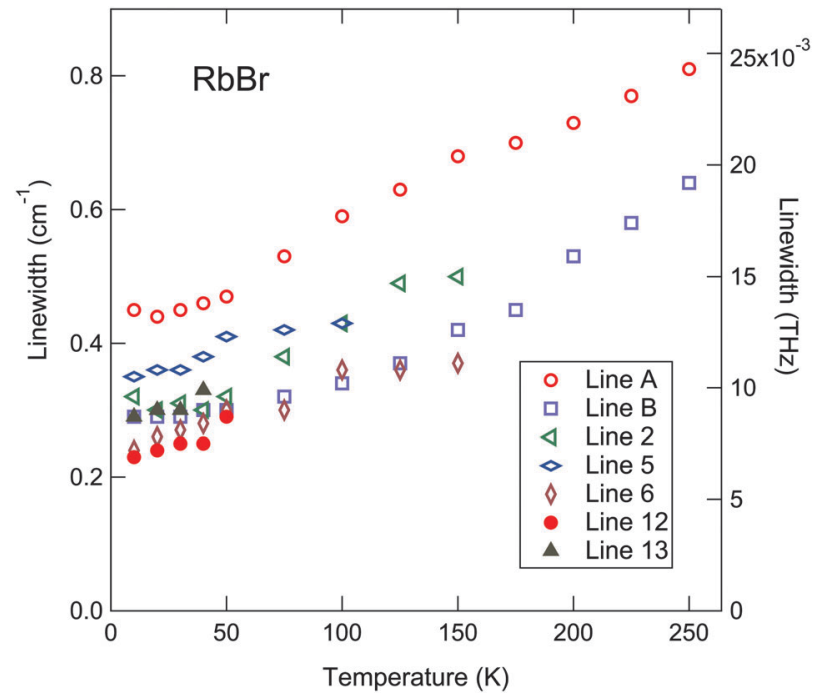

Fig. 11 Change of the linewidth of the libration lines with temperature, for the lines with a weak temperature dependence of the linewidth.

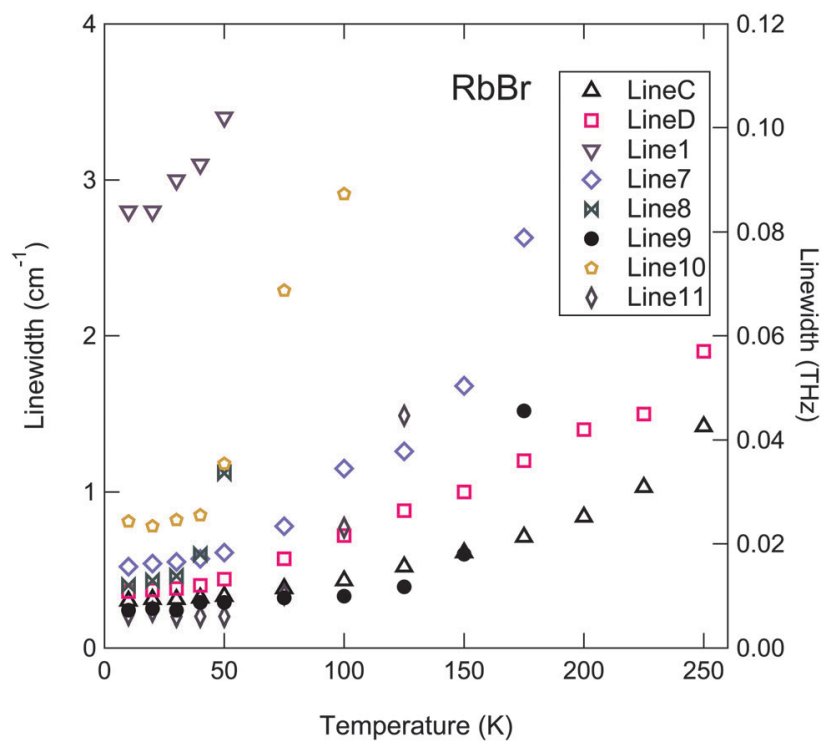

Fig. 12 Change of the linewidth of the libration lines with temperature, for some of the lines with a strong temperature dependence of the linewidth.

water is weakly bound to a crystal lattice and exhibits orientational disorder. ${ }^{1,51}$ Instead, $\Gamma(T)$ can be fitted well with eqn (1), with the quadratic term being substantial. The values of $\Gamma_{0}$, $a$ and $b$ are given in Table 2 . Non-negligible values of $b$ imply that the quartic anharmonic terms in the crystal potential are significant for these librations. ${ }^{24}$

Line intensity changes little with temperature for the lowermost temperatures (Fig. 13). It decreases with temperature more strongly above a characteristic temperature. This temperature is about $50 \mathrm{~K}$ for the lines observable at room temperature (A-D), while it is smaller for the other lines. Table 2 gives the rate of the temperature change of intensity in the linear approximation. The lines with strong temperature dependence of intensity are

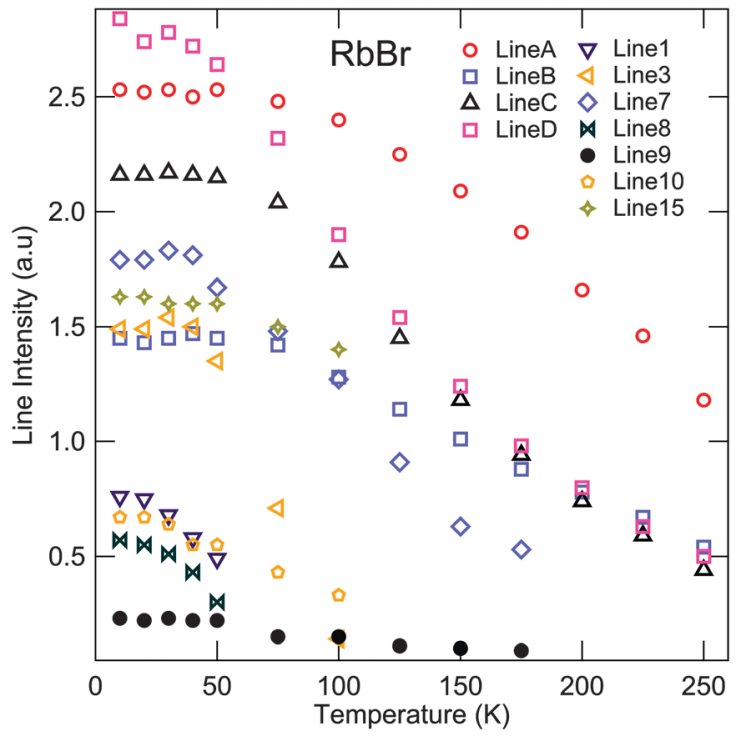

Fig. 13 Change of the line intensity for some of the libration lines with temperature.

1, 3, 7, D, and C. Lines B, 5, 9, 11, 14, and 15 have a weak temperature dependence of intensity, for example.

There seems to be a general correspondence between the lines with strong or weak temperature dependence of their position, linewidth and intensity. For example, lines 7 and $\mathrm{C}$ have a strong temperature dependence of their position, as well as linewidth and intensity (Fig. 10, 12 and 13). However, exceptions always occur when trying to group all the lines in this manner. For example, line $\mathrm{D}$ has a weak temperature dependence of absorption energy (Fig. 10), but strong temperature dependence of linewidth (Fig. 12) and intensity (Fig. 13).

Several of the absorption lines calculated by the PBE0 method can be identified in the observed spectrum (Fig. 9). These lines were used to distinguish the libration modes that lead to a strong or weak temperature dependence of linewidth. The librations did not consist of pure wagging, twisting or rocking modes. The type of librational coupling between the water molecules seemed to be a more important factor. We studied the distributions of the amplitudes and phases of libration for the 6 water molecules in our PBE0 modelling. For example, lines A and B with weak $\Gamma(T)$ consisted of correlated librations of all 6 water molecules, with large amplitudes. On the other hand, lines C, D and 15, all with strongly changing $\Gamma(T)$, are produced by collective librations where only some water molecules in the $\mathrm{RbBr}$ unit cell move with large amplitudes. However, lines 1 and 7, with strongly changing $\Gamma(T)$, do not follow this trend. The phase of these librations also plays a role in determining $\Gamma(T)$. Fully symmetric librations for lines A and B indicate that a weak $\Gamma(T)$ favours a fully symmetrical in-phase correlation between neighbouring water librations. All identified lines in Fig. 9 with strongly changing $\Gamma(T)$ had at least some of the neighbouring water pairs librating out of phase. Nevertheless, neither the phase of librations, distribution of amplitudes of water molecules in a unit $\mathrm{RbBr}$ cell, nor type of librations fully determine $\Gamma(T)$ by itself, either for weak or for strong $\Gamma(T)$ dependence. The combination 
of all these factors needs to be taken into account and we could not identify a large enough number of librational lines between modelling and experiment to find the exact combinations for each case. Distinguishing the libration modes for the lines with large and small values of $b$ was also not straightforward. Similar trends were obtained in regard to the temperature dependence of position and intensity for these lines.

\section{Conclusions}

Terahertz spectroscopy of hydrated $\mathrm{RbBr}$ reveals numerous vibrational and librational lines of water. Four distinct libration lines were observed at room temperature. Measurements at $10 \mathrm{~K}$ reveal an additional 15 librational lines. Numerical modelling of the spectrum using PBE gradient-corrected DFT confirmed that the absorption lines observed between 16.8 and $19.8 \mathrm{THz}$ (560 and $660 \mathrm{~cm}^{-1}$ ) are due to the water libration in $\mathrm{RbBr}$ crystal lattice. The hybrid form of the same functional, PBE0, helped identify 7 of the observed lines in this spectral range. All of these lines are produced by correlated librations of water molecules. The detailed temperature dependence of the spectrum shows that the librational lines red-shift with temperature. Most lines shift little with temperature below about $50 \mathrm{~K}$. Line shifts were linear with temperature for $T>50 \mathrm{~K}$, mostly at rates between 60 and $90 \mathrm{MHz} \mathrm{K}^{-1}$ (between (2 and 3) $\times 10^{-3} \mathrm{~cm}^{-1} \mathrm{~K}^{-1}$ ). However, two lines shifted much more rapidly with temperature. The temperature dependence of the linewidth for the librational lines gives two distinct groups of the lines. The linewidth changes linearly with temperature for the first group, with a rate of less than $60 \mathrm{MHz} \mathrm{K} \mathrm{K}^{-1}\left(2 \times 10^{-3} \mathrm{~cm}^{-1} \mathrm{~K}^{-1}\right)$. Cubic anharmonic terms in the crystal field are significant for the librations in this group. The linewidth changes much faster for the second group, typically quadratically in temperature. This shows that a quartic term in the anharmonic crystal field is significant for the librations in this group. A weak temperature dependence of linewidth seems to favour fully symmetric librations between the neighbouring water molecules. However, a full description of $\Gamma(T)$ would also need to take into account the distribution of libration amplitudes of waters in a unit $\mathrm{RbBr}$ cell, as well as the distribution of the libration types. The large number of librational lines in the spectrum shows that water loses its $C_{2 \mathrm{v}}$ symmetry in the RbBr crystal.

\section{Acknowledgements}

This work was supported by the Australian Research Council and Botswana International University of Science and Technology. We would like to thank University of Wollongong Information Technology Services for computing time on the UOW High Performance Computing Cluster. We thank John Mabon for assistance with the spectrum of $\mathrm{Rb}_{2} \mathrm{CO}_{3}$. Zhenguo Huang helped with thermogravimetric/mass spectrometer measurements.

\section{References}

1 V. P. Tayal, B. K. Srivastava, D. P. Khandelwal and H. D. Bist, Appl. Spectrosc. Rev., 1980, 16, 43-134.

2 D. M. Adams and P. J. Lock, J. Chem. Soc. A, 1971, 2801-2806.
3 S. Ebbinghaus, S. J. Kim, M. Heyden, X. Yu, U. Heugen, M. Gruebele, D. M. Leitner and M. Havenith, Proc. Natl. Acad. Sci. U. S. A., 2007, 104, 20749-20752.

4 F. Franks, Biophys. Chem., 2002, 96, 117-127.

5 M. Chaplin, Nat. Rev. Mol. Cell Biol., 2006, 7, 861-866.

6 D. V. Matyushov and A. Y. Morozov, Phys. Rev. E: Stat., Nonlinear, Soft Matter Phys., 2011, 84, 011908.

7 J. W. Lynn, Q. Huang, C. M. Brown, V. L. Miller, M. L. Foo, R. E. Schaak, C. Y. Jones, E. A. Mackey and R. J. Cava, Phys. Rev. B: Condens. Matter Mater. Phys., 2003, 68, 214516.

8 F. Despa, A. Fernandez and R. S. Berry, Phys. Rev. Lett., 2004, 93, 228104.

9 T. Ishiyama, H. Takahashi and A. Morita, Phys. Rev. B: Condens. Matter Mater. Phys., 2012, 86, 035408.

10 L. Zhi, G. Zhao, L. Guo and Q. Jing, Phys. Rev. B: Condens. Matter Mater. Phys., 2008, 77, 235435.

11 S. Haq, C. Clay, G. R. Darling, G. Zimbitas and A. Hodgson, Phys. Rev. B: Condens. Matter Mater. Phys., 2006, 73, 115414.

12 M. F. Kimmit, Far-Infrared Techniques, Pion Limited, 1970.

13 E. Brundermann, H.-W. Hübers and M. F. Kimmit, Terahertz Techniques, Springer, 2012.

14 S. D. Ganichev and W. Prettl, Intense Terahertz Excitation of Semiconductors, Oxford University Press, 2006.

15 R. A. Lewis, Terahertz Physics, Cambridge University Press, 2012.

16 L. R. Blaine, J. Res. Natl. Bur. Stand., Sect. C, 1963, 67C, 207-215.

17 K. Teegarden and G. Baldini, Phys. Rev., 1967, 155, 896-907.

18 J. E. Eby, K. J. Teegarden and D. B. Dutton, Phys. Rev., 1959, 116, 1099-1105.

19 D. McCarthy, Appl. Opt., 1968, 7, 2221-2225.

20 B. S. Ault, J. Am. Chem. Soc., 1978, 100, 2426-2433.

21 J.-J. Max and C. Chapados, J. Chem. Phys., 2001, 115, 2664-2675.

22 J. van der Elsken and D. W. Robinson, Spectrochim. Acta, 1961, 17, 1249-1256.

23 H. Lutz and H. Christian, J. Mol. Struct., 1983, 101, 199-212.

24 I. P. Ipatova, A. A. Maradudin and R. F. Wallis, Phys. Rev., 1967, 155, 882-895.

25 F. Neese, Wiley Interdiscip. Rev.: Comput. Mol. Sci., 2012, 2, 73-78.

26 J. P. Perdew, K. Burke and M. Ernzerhof, Phys. Rev. Lett., 1996, 77, 3865-3868.

27 M. Ernzerhof and G. E. Scuseria, J. Chem. Phys., 1999, 110, 5029-5036.

28 S. Grimme, J. Comput. Chem., 2006, 27, 1787-1799.

29 A. Schäfer, H. Horn and R. Ahlrichs, J. Chem. Phys., 1992, 97, 2571-2577.

30 R. Krishnan, J. S. Binkley, R. Seeger and J. A. Pople, J. Chem. Phys., 1980, 72, 650-654.

31 L. A. Curtiss, M. P. McGrath, J.-P. Blaudeau, N. E. Davis, J. Robert, C. Binning and L. Radom, J. Chem. Phys., 1995, 103, 6104-6113.

32 The Ahlrichs (2df,2pd) polarisation functions were obtained from the TurboMole basis set library under ftp.chemie.unikarlsruhe.de/pub/basen. 
33 W. Humphrey, A. Dalke and K. Schulten, J. Mol. Graphics, 1996, 14, 33-38.

34 V. N. Staroverov, G. E. Scuseria, J. Tao and J. P. Perdew, J. Chem. Phys., 2003, 119, 12129-12137.

35 C. Medcraft, D. McNaughton, C. D. Thompson, D. R. T. Appadoo, S. Bauerecker and E. G. Robertson, Phys. Chem. Chem. Phys., 2013, 15, 3630-3639.

36 S. A. Deshmukh and S. K. R. S. Sankaranarayanan, Phys. Chem. Chem. Phys., 2012, 14, 15593-15605.

37 S. Le Caer, S. Pin, S. Esnouf, Q. Raffy, J. P. Renault, J.-B. Brubach, G. Creff and P. Roy, Phys. Chem. Chem. Phys., 2011, 13, 17658-17666.

38 http://www.chemicalbook.com/SpectrumEN/584-09-8/IR1.htm.

39 W. C. Price, W. F. Sherman and G. R. Wilkinson, Proc. $R$. Soc. London, Ser. A, 1958, 247, 467-468.

40 J.-J. Max and C. Chapados, J. Chem. Phys., 2009, 131, 184505.

41 J.-J. Max and C. Chapados, J. Chem. Phys., 2002, 116, 4626-4642.

42 A. Millo, Y. Raichlin and A. Katzir, Appl. Spectrosc., 2005, 59, 460-466.
43 A. B. McCoy, T. L. Guasco, C. M. Leavitt, S. G. Olesen and M. A. Johnson, Phys. Chem. Chem. Phys., 2012, 14, 7205-7214.

44 A. T. Kellogg and R. O. Pohl, J. Appl. Phys., 1964, 35, 1359-1360.

45 G. Ferraris and M. Franchini-Angela, Acta Crystallogr., Sect. B: Struct. Crystallogr. Cryst. Chem., 1972, 28, 3572-3583.

46 W. J. Hehre, L. Radom, P. Schleyer and J. A. Pople, Ab initio molecular orbital theory, Wiley, 1986.

47 I. M. Alecu, J. Zheng, Y. Zhao and D. G. Truhlar, J. Chem. Theory Comput., 2010, 6, 2872-2887.

48 W. S. Benedict, N. Gailar and E. K. Plyler, J. Chem. Phys., 1956, 24, 1139-1165.

49 C. Liu, W. Li and W. Wang, Phys. Rev. E: Stat., Nonlinear, Soft Matter Phys., 2013, 87, 052309.

50 J. J. Loparo, C. J. Fecko, J. D. Eaves, S. T. Roberts and A. Tokmakoff, Phys. Rev. B: Condens. Matter Mater. Phys., 2004, 70, 180201.

51 J. J. Rush, P. S. Leung and T. I. Taylor, J. Chem. Phys., 1966, 45, 1312-1317. 